\title{
Dynamic remodeling of the plastid envelope membranes - a tool for chloroplast envelope in vivo localizations
}

\section{Frederique K. H. Breuers ${ }^{1 \dagger}$, Andrea Bräutigam ${ }^{1 \dagger}$, Stefan Geimer ${ }^{2 \dagger}$, Ulla Y. Welzel ${ }^{2}$, Giovanni Stefano ${ }^{3}$, Luciana Renna ${ }^{3}$, Federica Brandizzi ${ }^{3}$ and Andreas P. M. Weber ${ }^{1 *}$}

1 Institute for Plant Biochemistry, Heinrich Heine University Düsseldorf, Düsseldorf, Germany

2 Electron Microscopy Laboratory, Institute for Cell Biology, University of Bayreuth, Bayreuth, Germany

${ }^{3}$ Plant Research Laboratory, Department of Energy, Michigan State University, East Lansing, MI, USA

\section{Edited by:}

Patrick Xuechun Zhao, Samuel

Roberts Noble Foundation, USA

Reviewed by:

Vagner Benedito, West Virginia

University, USA

Haiquan Li, University of Illinois at

Chicago, USA

\section{*Correspondence:}

Andreas P. M. Weber, Institute for

Plant Biochemistry, Heinrich Heine University, Geb. 26.03.01,

Universitätsstrasse 1, D-40225

Düsseldorf, Germany.

e-mail: andreas.weber@uni-

duesseldorf.de

${ }^{\dagger}$ Frederique K. H. Breuers, Andrea Bräutigam and Stefan Geimer have contributed equally to this work.
Two envelope membranes delimit plastids, the defining organelles of plant cells. The inner and outer envelope membranes are unique in their protein and lipid composition. Several studies have attempted to establish the proteome of these two membranes; however, differentiating between them is difficult due to their close proximity. Here, we describe a novel approach to distinguish the localization of proteins between the two membranes using a straightforward approach based on live cell imaging coupled with transient expression. We base our approach on analyses of the distribution of GFP-fusions, which were aimed to verify outer envelope membrane proteomics data. To distinguish between outer envelope and inner envelope protein localization, we used AtTOC64-GFP and AtTIC40-GFP, as respective controls. During our analyses, we observed membrane proliferations and loss of chloroplast shape in conditions of protein over-expression. The morphology of the proliferations varied in correlation with the suborganellar distribution of the over-expressed proteins. In particular, while layers of membranes built up in the inner envelope membrane, the outer envelope formed long extensions into the cytosol. Using electron microscopy, we showed that these extensions were stromules, a dynamic feature of plastids. Since the behavior of the membranes is different and is related to the protein localization, we propose that in vivo studies based on the analysis of morphological differences of the membranes can be used to distinguish between inner and outer envelope localizations of proteins. To demonstrate the applicability of this approach, we demonstrated the localization of AtLACS9 to the outer envelope membrane. We also discuss protein impact on membrane behavior and regulation of protein insertion into membranes, and provide new hypotheses on the formation of stromules.

Keywords: chloroplast envelope, membrane proliferation, membrane protein, outer envelope membrane, inner envelope membrane, LACS9, stromule

\section{INTRODUCTION}

The defining organelle of plant cells is the plastid. Plant plastids derive from a single primary endosymbiosis event, and are delimited by an outer and an inner envelope membrane. These membranes are the gateway for protein (Strittmatter et al., 2010), lipid (Benning, 2009), and metabolite flux (Linka and Weber, 2010; Breuers et al., 2011; Facchinelli and Weber, 2011; Weber and Linka, 2011) between plastid and cytosol. Further, the membranes are the location of several biosynthetic processes, like membrane lipid biosynthesis (Benning, 2009). During the past decade, several proteomics analyses (e.g., Ferro et al., 2003, 2010; Rolland et al., 2003; Bräutigam et al., 2008a,b; Bräutigam and Weber, 2009; Sun et al., 2009; Joyard et al., 2010) contributed to a better understanding of the protein composition of the envelope membranes and thus provided new platforms to increase knowledge on the functions and evolutionary role of these permeable barriers.
In the last decades, the concept of the chloroplast envelope membranes has changed from a static border to a highly dynamic interface between the plastids and the cytosol. For example, thin, stroma filled structures surrounded by both envelope membranes have been observed extruding from the plastid under natural conditions in a wide variety of plant species (Gunning, 2005; Holzinger et al., 2007a,b; Sage and Sage, 2009). Further, they have been found in plant cells transformed with constructs encoding stroma-targeted and envelope GFP-fusion proteins (Köhler et al., 1997a,b; Tirlapur et al., 1999; Köhler and Hanson, 2000; Shiina et al., 2000; Arimura et al., 2001; Pyke and Howells, 2002; Hanson and Sattarzadeh, 2008; Shaw and Gray, 2011). These structures are called stromules, which stands for stroma filled tubules (Köhler et al., 1997a,b; Köhler and Hanson, 2000; Hanson and Köhler, 2001). Several functions have been proposed for stromules, such as increasing surface area, connecting chloroplasts (and possibly other organelles), a role in signaling pathways, and 
adaption to temperature and light stress (for a recent review: Hanson and Sattarzadeh, 2011). Despite the observation of stromules in non-transformed plant cells via light and electron microscopy, GFP-fusions have remained the predominant tool for observing stromule formation in vivo.

The localization of plastid proteins to distinct subcompartments of the chloroplast, such as stroma, envelope membranes, and thylakoids, via GFP-fusion proteins is challenging for several reasons. Stromal proteins are relatively easy to distinguish from envelope localizations, because envelope localized proteins form a ring like structure around the chloroplast. In contrast, distinguishing between localization in the outer envelope or inner envelope is challenging due to their close proximity. Here, we demonstrate that a combination of membrane dynamics and GFP-fusion proteins provides an efficient tool for distinguishing between inner and outer envelope proteins, using the long chain fatty acid CoA synthetase AtLACS9 as an example. AtLACS9 is the only investigated protein with LACS activity in the chloroplast and has been shown to account for $90 \%$ of the LACS activity in the chloroplast (Schnurr et al., 2002; Shockey et al., 2002); however, its location has not been conclusively demonstrated to date. While LACS activity has been described in outer envelopes of spinach chloroplasts (Roughan and Slack, 1977), the in vivo LACS9 was non-specifically described as envelope localized (Schnurr et al., 2002; Koo et al., 2004; Sun et al., 2009; Zhao et al., 2010).

\section{MATERIALS AND METHODS PLANT GROWTH CONDITION}

Nicotiana benthamiana plants were grown for 4-6weeks in a green house, while Nicotiana tabacum plants were grown for 46 weeks in a growth chamber using a $16 \mathrm{~h} / 8 \mathrm{~h}$ day/night cycle with temperatures of $27^{\circ} \mathrm{C} / 24^{\circ} \mathrm{C}$.

\section{CLONING}

The genes for AtTOC64-III (At3g17970), AtLACS9 (At1g77590), AtTic40 (At5g16620), AtTPT (At5g46110), AtAPG1 (At3g63410), and AtLrgB (At1g32080) were amplified from cDNA and cloned into the plant expression vector pMDC83 (Curtis and Grossniklaus, 2003) for C-terminal GFP-fusion. In addition AtLACS9 was cloned into pUBC-GFP (Grefen et al., 2010) as Ubiquitin10 promoter driven construct in C-terminal GFP fusion, pABindGFP (Bleckmann et al., 2010) as $\beta$-estradiol inducible promoter driven construct in C-terminal GFP-fusion, and pMDC32 (Curtis and Grossniklaus, 2003) as untagged 35 S-promoter driven constructs.

\section{AGROBACTERIUM TUMEFACIENS PREPARATION}

Agrobacterium tumefaciens [GV3101(pMP90)] (Koncz and Schell, 1986) was transformed with the plasmids, and grown on LB plates $(5 \mathrm{~g}$ yeast extract, $10 \mathrm{~g}$ tryptone, $5 \mathrm{~g} \mathrm{NaCl}, 1 \mathrm{ml} 1 \mathrm{M}$ $\mathrm{NaOH}$ in $1 \mathrm{l}$ ) or YEB plates ( $1 \mathrm{~g}$ yeast extract, $5 \mathrm{~g}$ tryptone, $5 \mathrm{~g}$ beef extract, $5 \mathrm{~g}$ sucrose, $0.5 \mathrm{~g} \mathrm{MgSO}_{4} \times 7 \mathrm{H}_{2} \mathrm{O}$, in 11) containing rifampicin $(50-150 \mu \mathrm{g} / \mathrm{ml})$, gentamycin, $(25-50 \mu \mathrm{g} / \mathrm{ml})$, and either kanamycin $(25-50 \mu \mathrm{g} / \mathrm{ml}$; pMDC83 and pMDC32) or spectinomycin $(100 \mu \mathrm{g} / \mathrm{ml}$; pUBC-GFP and pABindGFP), depending on the plasmid resistance. Colonies were grown for $48 \mathrm{~h}$ in a $30^{\circ} \mathrm{C}$ chamber and used for transformation of Nicotiana leaves.

\section{AGROBACTERIUM TUMEFACIENS MEDIATED TRANSFORMATION OF N. TABACUM FOR IN PLANTA STUDIES}

Colonies were inoculated in $5 \mathrm{ml}$ liquid cultures of LB containing antibiotics (see above) and grown under constant shaking of $220 \mathrm{rpm}$ and $30^{\circ} \mathrm{C}$ in Innova incubation shakers in culture tubes for 16-24 h. A 1-ml aliquot of the cell cultures was harvested by centrifugation and re-suspended in fresh infiltration buffer [IF; $2 \mathrm{mM} \mathrm{Na} 3 \mathrm{PO}_{4}, 50 \mathrm{mM} \mathrm{MES} / \mathrm{KOH}$ ( $\mathrm{pH} 7.6$ ), $5 \mathrm{mg} / \mathrm{ml}$ glucose, $200 \mathrm{mM}$ acetosyringone]. Bacteria $\left(\mathrm{OD}_{600}=0.05\right)$ were used for infiltration into N. tabacum leaves, as described earlier (Batoko et al., 2000). After infiltration, all plants were kept in growth chamber under conditions of $16 \mathrm{~h} / 8 \mathrm{~h}$ day/night cycle with temperatures of $27^{\circ} \mathrm{C} / 24^{\circ} \mathrm{C}$.

\section{AGROBACTERIUM TUMEFACIENS MEDIATED TRANSFORMATION OF N. BENTHAMIANA FOR PROTOPLAST STUDIES}

Colonies were streaked-out on new plates and grown for additional $24 \mathrm{~h}$ in a $30^{\circ} \mathrm{C}$ chamber. Liquid cultures of YEB medium containing antibiotics (see above) of $12 \mathrm{ml}$ were grown under constant shaking of $220 \mathrm{rpm}$ and $30^{\circ} \mathrm{C}$ in Innova incubation shakers in $125 \mathrm{ml}$ Erlenmeyer flasks over night. Cells were harvested by centrifugation and re-suspended in fresh activation medium [containing $10 \mathrm{mM} \mathrm{MES/KOH} \mathrm{(pH} \mathrm{5.6),} 10 \mathrm{mM} \mathrm{MgCl}_{2}$, and $150 \mu \mathrm{M}$ acetosyringone]. Bacteria containing protein expression vectors $\left(\mathrm{OD}_{600}=0.4\right)$ were mixed together with a 19 helper strain $\left(\mathrm{OD}_{600}=0.3\right.$; Voinnet et al., 2003). The mixture was incubated for $2 \mathrm{~h}$ at room temperature and infiltrated into $N$. benthamiana leaves. After infiltration, all plants were kept in the greenhouse until the end of analysis (adapted from Wydro et al., 2006; Gehl et al., 2009).

\section{PROTOPLAST ISOLATION}

Four to seven leaf disks with a diameter of $0.8 \mathrm{~cm}$ of transfected $N$. benthamiana were cut with a cork borer and transferred into a 10$\mathrm{ml}$ syringe containing $2 \mathrm{ml}$ of cell wall digestion solution (CWDS; $1.5 \%$ cellulase R-10, $0.4 \%$ macerozyme R-10, $0.4 \mathrm{M}$ mannitol, $20 \mathrm{mM} \mathrm{KCl}, 20 \mathrm{mM}$ MES (pH 5.6), $10 \mathrm{mM} \mathrm{CaCl}_{2}, 0.1 \%$ BSA). The enzyme solution was infiltrated into the intercellular space of the leaf disks. Leaf disks were than transferred to a 2-ml Eppendorf tube with the enzyme solution and incubated for $2-4 \mathrm{~h}$ at $20-25^{\circ} \mathrm{C}$. Protoplasts were shaken out of the digested tissue and pelleted by gravity upon removal of undigested tissue with a pipette tip. CWDS was exchanged by minus-enzymes-solution (-ES; $0.4 \mathrm{M}$ mannitol, $20 \mathrm{mM} \mathrm{KCl}, 20 \mathrm{mM}$ MES (pH 5.6), $10 \mathrm{mM} \mathrm{CaCl}_{2}, 0.1 \%$ BSA) and used to wash the isolated protoplasts (adapted from Yoo et al., 2007).

\section{MICROSCOPIC ANALYSIS}

Protoplasts and plant tissue were analyzed by an inverted or an upright Zeiss LSM 510 META confocal laser-scanning microscope. GFP and chlorophyll were excited by the 488 -nm laser line of an Argon laser and the emission was collected at 505-550 nm and at $>650 \mathrm{~nm}$, respectively. Differential interference contrast (DIC) microscopic analysis was done via a Nikon Eclipse T inverse microscope. Pictures were taken via a b/w-ProgResMF ${ }^{\mathrm{TM}}$-camera by Jenoptik. 


\section{TRANSMISSION ELECTRON MICROSCOPY}

Leaf pieces (about $1-2 \mathrm{~mm}^{2}$ ) were cut from infiltrated N. benthamiana leaves and incubated in fixation buffer $(2.5 \%$ glutaraldehyde and $3 \%$ formaldehyde in $50 \mathrm{mM}$ sodium cacodylate, $\mathrm{pH} 7.3$ ) at room temperature and a pressure of $20 \mathrm{mbar}$ for $60 \mathrm{~min}$. The fixation buffer was replaced, and the samples were fixed overnight at $4^{\circ} \mathrm{C}$. After three 10 -min washes in distilled water, the samples were osmicated in $1 \%$ osmium tetroxide (in distilled water) for $60-100 \mathrm{~min}$ at $4^{\circ} \mathrm{C}$, rinsed three times for $10 \mathrm{~min}$ each in distilled water and incubated in $1 \%$ uranyl acetate (in distilled water) at $4^{\circ} \mathrm{C}$ overnight. After three washes of $10 \mathrm{~min}$ each in distilled water the samples were embedded in 1\% Difco ${ }^{\mathrm{TM}}$ Agar noble (Becton, Dickinson and Company, Sparks, MD, USA), dehydrated using increasing concentrations of ethanol and embedded in glycidyl ether 100 (formerly Epon 812; Serva, Heidelberg, Germany) with propylene oxide as intermediate solvent following standard procedures. Polymerization was carried out for $48 \mathrm{~h}$ at $65^{\circ} \mathrm{C}$. Ultrathin sections $(\sim 60 \mathrm{~nm})$ were cut with a diamond knife (type ultra $35^{\circ}$; Diatome, Biel, Suisse) on an EM UC6 ultramicrotome (Leica Microsystems, Wetzlar, Germany) and mounted on single-slot Pioloform-coated copper grids (Plano, Wetzlar, Germany). The sections were stained with uranyl acetate and lead citrate (Reynolds, 1963) and viewed with a JEM-2100 transmission electron microscope (JEOL, Tokyo, Japan) operated at $80 \mathrm{kV}$. Micrographs were taken using a $4080 \times 4080$ or $1350 \times 1040$ pixels charge-coupled device camera (UltraScan 4000 or Erlangshen ES500W respectively, Gatan, Pleasanton, CA, USA) and Gatan Digital Micrograph software (version 1.70.16). Image brightness and contrast were adjusted and figures assembled using Adobe Photoshop 8.0.1.

\section{RESULTS}

\section{DISTINCT STRUCTURES ARE OBSERVED WHEN EXPRESSING ATTOC64} AND ATTIC4O IN TRANSIENTLY TRANSFORMED TOBACCO CELLS

To examine labeling patterns of inner and outer envelope membrane proteins, we performed in vivo localization studies using fluorescent-tagged fusion proteins in $N$. benthamiana. Since it is known that an $\mathrm{N}$-terminal transit peptide, or at least a signal sequence, is needed to insert envelope proteins into the membrane (Bionda et al., 2010), we fused the fluorescent tag to the C-terminus of the proteins of interest. As a control for the outer envelope, we used AtTOC64-III, a co-receptor and co-chaperone of the TOC (Translocon of the outer chloroplast membrane) complex (Sohrt and Soll, 2000; Qbadou et al., 2007). As a control for the inner envelope, we used AtTIC40, a component of the TIC (Translocon of the inner chloroplast membrane) complex (Chou, 2003; Singh et al., 2008). As cytosolic control we expressed GFP driven by a $35 \mathrm{~S}$-promoter (Figure 1A).

By analyzing the distribution of 35S:AtTOC64-GFP, we detected GFP signal in bright circular structures surrounding the chlorophyll autofluorescence (Figure 1B). This distribution is consistent with previous observations by Bae et al. (2008) and with that of other outer envelope proteins such as CHUP1 (e.g., Oikawa et al., 2003, 2008; Schmidt Von Braun and Schleiff, 2008) and OEP7 (Lee et al., 2001). However, we also observed GFP labeled extensions looping out from the envelope circles (Figure 1B).

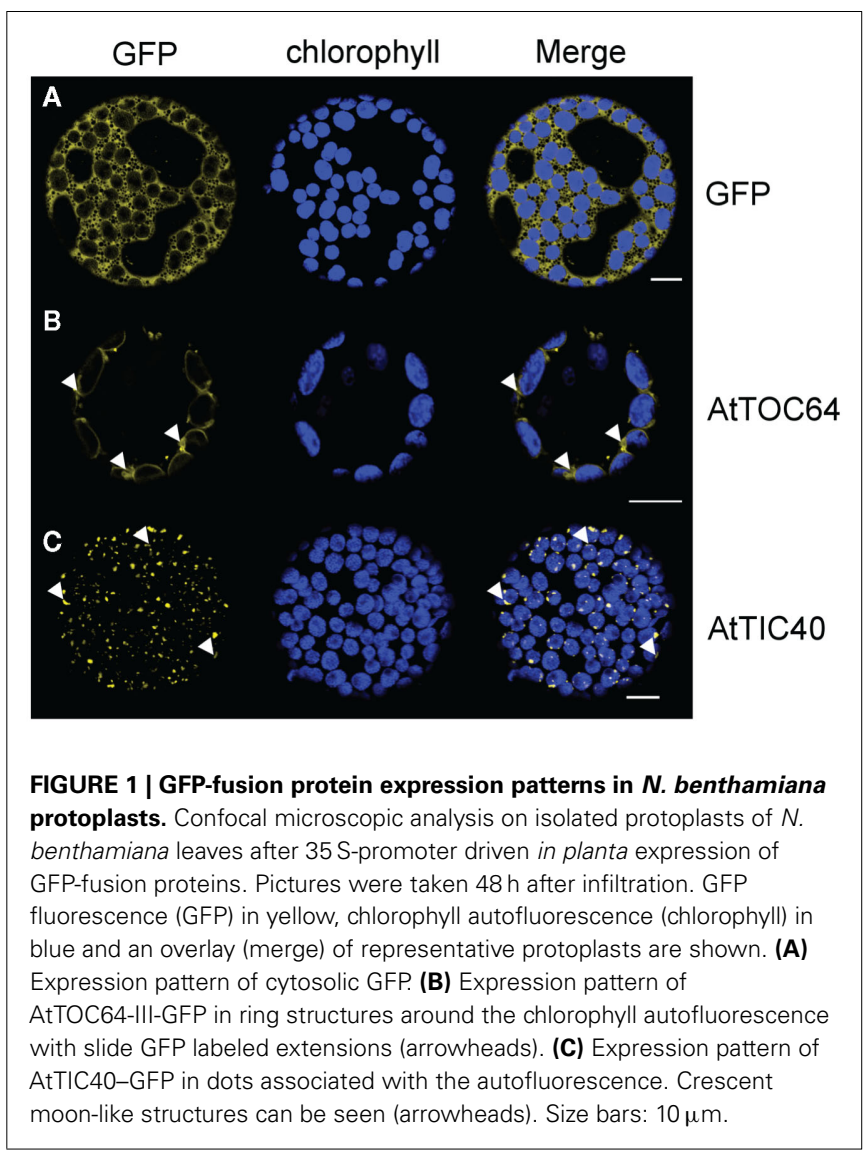

Next we analyzed the distribution of 35S:AtTIC40-GFP and observed GFP labeling patterns. The GFP signal was clearly distinct from that of AtTOC64-GFP. We detected GFP as small dots and crescent moon-like structures associated with the chlorophyll autofluorescence (Figure 1C). In addition to these most frequently observed patterns, some protoplasts expressed GFP in circles around the chloroplast. Filamentous extrusions from the chloroplast were never observed. We hypothesized that the observed differences between the labeling patterns might be due to different expression levels within the transient expression system employed in our study. To test this hypothesis, we conducted a time course analysis of the expression pattern after introduction of the tagged proteins into transiently transformed cells.

\section{ABERRANT MEMBRANE STRUCTURE FORMATION IS DEPENDENT ON THE PROTEIN AMOUNT}

Agrobacterium mediated transient transfection of plant cells is based on the insertion of T-DNA into the cell genome. Afterward, protein is constantly synthesized and thus protein concentration is increasing in a time dependent manner (Wydro et al., 2006). Hence, when our constructs were inserted into the cell genome, we were able to observe different protein loading in the respective membrane at different time points. AtTIC40-GFP and AtTOC64-GFP expression were monitored 48, 72, and $96 \mathrm{~h}$ after leaf infiltration. We took pictures of representative expression patterns for each time point and each protein. At least 50 transfected protoplasts were evaluated for each sample and the experiments 
were done at least three times independently for each expressed protein.

Fortyeight hours after infiltration with Agrobacterium with AtTIC40-GFP the expression pattern was congruent with the observations outlined above (Figure 2A). In addition to GFP dots the crescent moon structures were highly abundant in the transfected protoplasts (Figure 2A zoom). In protoplasts isolated from leaves at 3 days after infiltration, a circular pattern surrounding the chloroplast dominated the protoplast samples (Figure 2B). However, most of the circles were incomplete and surrounded no more than half of the autofluorescence. Small areas between such half circles remained free of GFP signal (Figure 2B zoom). After an additional $24 \mathrm{~h}$, we observed full circles (Figure 2C). Most of the protoplasts also contained minor loop-like structures evolving from the circles (Figure 2C zoom).

After 2 days expressing 35S:AtTOC64-GFP, we observed chlorophyll autofluorescence surrounded by circular GFP fluorescence; most likely illuminating the predominant shape of the outer envelope membrane (Figure 3). However, some protoplasts already contained labeled loop-like deformations (Figure 3A zoom). These deformations became more abundant when we expressed AtTOC64-GFP for an additional $24 \mathrm{~h}$. We observed various labeled thin tubular structures evolving from the chloroplastsurrounding circles that partially connected different chloroplasts (Figure 3B). These structures became even more abundant in the

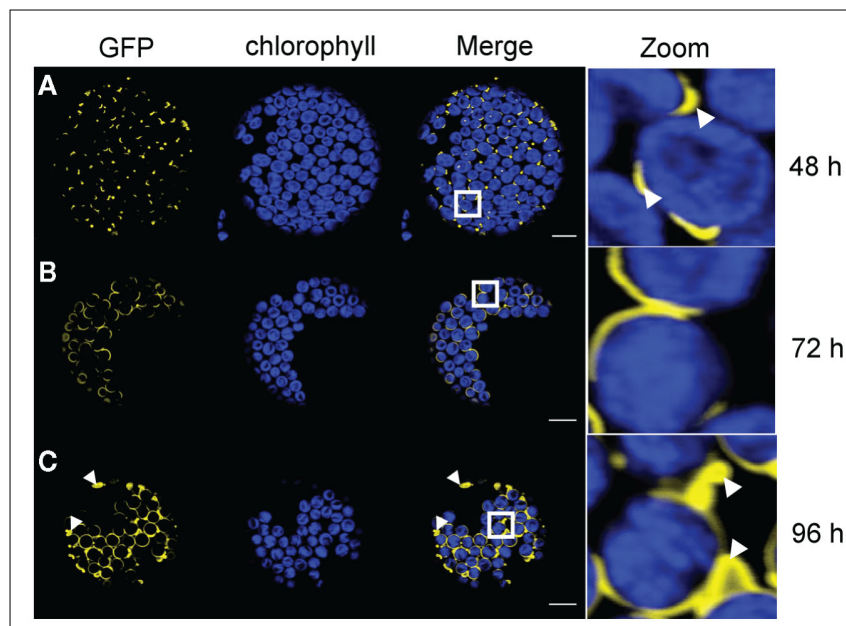

FIGURE 2 |Time course analysis of expression patterns for AtTIC40-GFP in $\boldsymbol{N}$. benthamiana protoplasts. Time based confocal microscopic analysis on isolated protoplasts of $N$. benthamiana leaves after 35 S-promoter driven in planta expression of AtTIC40-GFP. GFP fluorescence (GFP) in yellow, chlorophyll autofluorescence (chlorophyll) in blue, an overlay (merge) and a magnification (zoom) of representative protoplasts are shown. The white box indicates the merged region magnified under zoom. (A) Expression pattern 2 days (48 h) after Agrobacterium infiltration shows short crescent moon-like structures (arrowheads) and dots associated with the chloroplasts. GFP signal slightly departs from chlorophyll autofluorescence. (B) Expression pattern 3 days (72 h) after Agrobacterium infiltration displays half circles around the chloroplasts. (C) Expression pattern 4 days (96 h) after Agrobacterium infiltration shows partially full circles surrounding the chloroplasts, interrupted circles, and scattered extensions (arrowheads). Size bars: $10 \mu \mathrm{m}$. protoplasts after $96 \mathrm{~h}$ of expression (Figure 3C). A network of thin tubules could be detected protruding through the entire cell. We observed labeled tubules of up to $40 \mu \mathrm{m}$ spanning through the protoplast and connecting individual chloroplasts with each other (Figures 3B,C zoom).

The structures observed after expressing the inner envelope protein AtTIC40 or outer envelope protein AtTOC64 indicated that the membranes were altered. We hypothesized that these alteration were due to the high protein amounts produced in the transiently transformed cells and not due to the intrinsic properties of the expressed proteins.

\section{INNER MEMBRANE ALTERATION IS INDEPENDENT OF THE PROTEIN PROPERTIES}

To test this hypothesis, we designed fluorophore expression vectors for additional inner membrane proteins, such as AtTPT, the triosephosphate carrier in the inner envelope membrane (Fischer et al., 1994); AtAPG1, the membrane-associated protein catalyzing the methylation of demethylplastoquinol to plastoquinone-9 (Dreses-Werringloer et al., 1991; Motohashi et al., 2003); and AtL$\mathrm{rgB}$, a highly abundant protein of unknown molecular function (Yang et al., 2012). In all cases the observed patterns were similar to those observed with AtTIC40-GFP, punctate structures around the chloroplast (Figure 4A), crescent moon structures (Figure 4B) and out loops (Figure 4C). We thus conclude that the observed

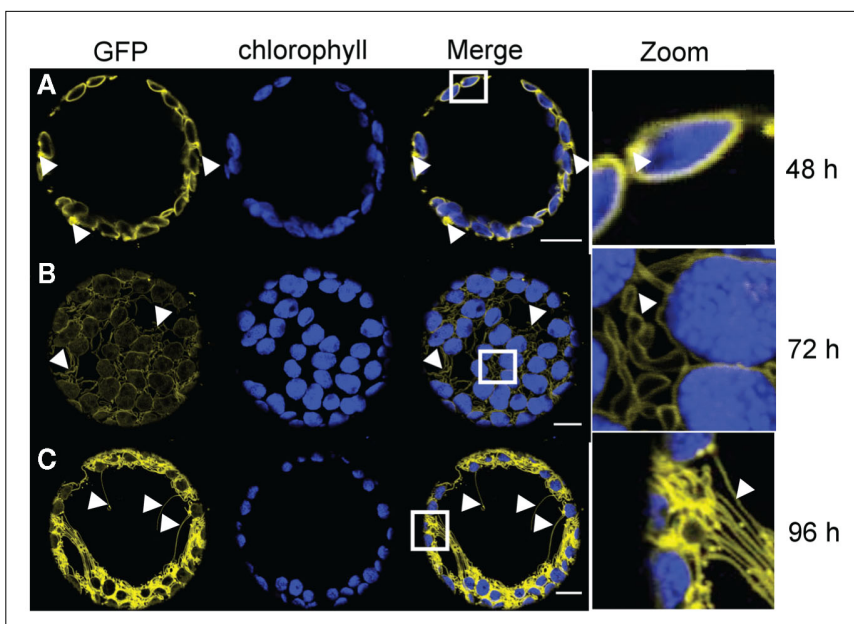

FIGURE 3 |Time course analysis of expression patterns for AtTOC64-III-GFP in $\boldsymbol{N}$. benthamiana protoplasts. Time based confocal microscopic analysis on isolated protoplasts of $N$. benthamiana leaves after 35 S-promoter driven in planta expression of AtTOC64-III-GFP. GFP fluorescence (GFP) in yellow, chlorophyll autofluorescence (chlorophyll) in blue, an overlay (merge) and a magnification (zoom) of representative protoplasts are shown. The white box indicates the merged region magnified under zoom. (A) Expression pattern 2 days (48h) after Agrobacterium infiltration shows ring structures surrounding the chloroplasts with slight extensions proliferating from the ring. (B) Expression pattern 3 days (72 h) after Agrobacterium infiltration displays along the chloroplast associated circles extensions of different length. Extensions are connecting the chloroplasts with each other. (C) Expression pattern 4 days (96 h) after Agrobacterium infiltration shows a network of GFP labeled structures surrounding the chloroplasts and spanning though the entire protoplasts. Size bars: $10 \mu \mathrm{m}$. 
membrane deformations are independent of the function and the biochemical properties of the inner envelope proteins but due to the increased protein amounts.

\section{LACS9 IS A CHLOROPLAST OUTER ENVELOPE PROTEIN}

To investigate to which of the envelope membrane layers AtLACS9 was targeted, we expressed 35S:AtLACS9-GFP in N. benthamiana leaves for subsequent protoplast isolation. After $48 \mathrm{~h}$ we observed circular structures around the chloroplast (Figure 5A), as previously seen for AtTOC64-III expression (Figure 2A). Also for AtLACS9, we saw small loops protruding into the cytosol (Figure 5A zoom). After $72 \mathrm{~h}$ of expression the majority of evaluated protoplasts showed increased amounts of loop structures and thin tubules arising form the chloroplast (Figure 5B). The expression pattern 4 days after infiltration was dominated by an extensive system of tubular structures connecting the GFP labeled circles around the chloroplast (Figure 5C). All these observations were congruent with the expression patterns previously observed for the outer envelope protein AtTOC64-III. We hence classified AtLACS9 as a chloroplast outer envelope protein.

\section{EXPERIMENTAL CONDITIONS DO NOT AFFECT THE MEMBRANE ALTERATION}

In all cases, we isolated protoplasts from transiently transformed tobacco leaves before microscopic observation of the GFP labeling

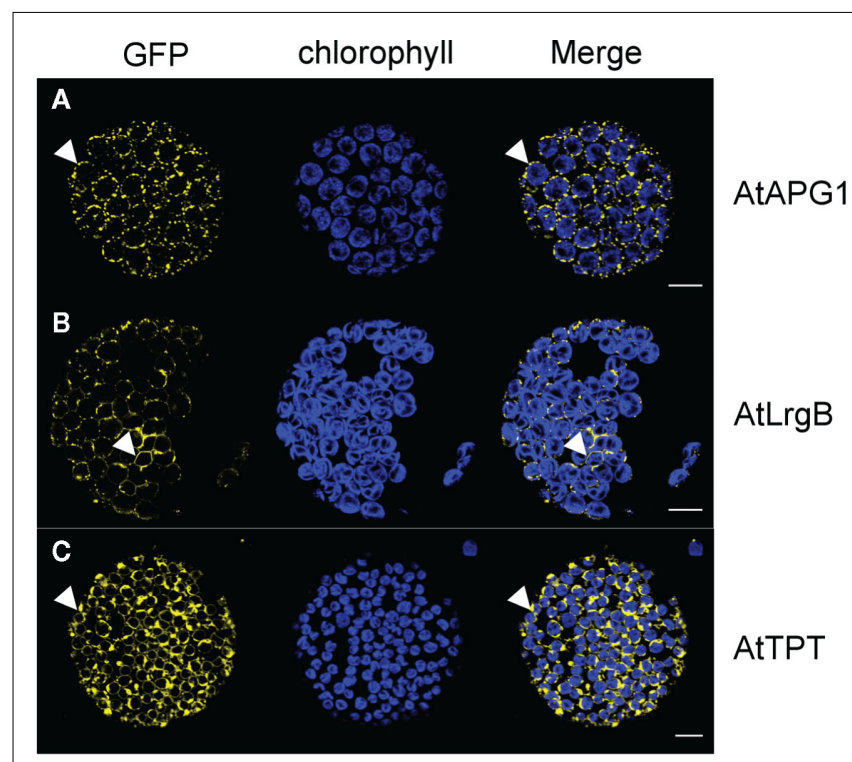

FIGURE 4 | GFP-fusion inner envelope protein expression patterns in $\boldsymbol{N}$. benthamiana protoplasts. Confocal microscopic analysis on isolated protoplasts of $N$. benthamiana leaves after 35 S-promoter driven in planta expression of GFP-fusion proteins. Pictures were taken $72 \mathrm{~h}$ after infiltration. GFP fluorescence (GFP) in yellow, chlorophyll autofluorescence (chlorophyll) in blue and an overlay (merge) of representative protoplasts are shown. (A) Expression pattern of AtAPG1-GFP in dot structures associated with the chloroplast. (B) Expression pattern of AtLrgB-GFP in interrupted ring structures around the chlorophyll autofluorescence. (C) Expression pattern of AtTPT-GFP in circle structures with slide extensions proliferating from the circle. Size bars: $10 \mu \mathrm{m}$. pattern. It was thus possible that the protoplast isolation procedure, involving cell wall digestions with cellulase and macerozyme might have influenced the observed patterns. To exclude a possible effect of cell wall digestion on the observed membrane alterations, we performed in planta analyses on the outer envelope protein AtLACS9 and the inner envelope protein AtTIC40. We chose N. tabacum as our model for these experiments and focused on expression in leaf epidermis cells. In the case of AtLACS9GFP we observed the formation of thin extensions arising from a GFP labeled plastids (Figure 6A), congruent with observations obtained with chloroplasts in protoplasts. This expression pattern differed from that of AtTIC40-GFP, which was expressed in dot like structures attached to the chloroplasts (Figure 6B). Also this observation was congruent with our findings in the protoplast assays. Therefore we conclude that the digestion of the cell wall during protoplast isolation was unlikely to affect the membrane alterations induced by expression of GFP-tagged proteins. Similarly, an effect of the p19 helper strain (Voinnet et al., 2003) used in the N. benthamiana transfection but not used in the N. tabacum plant, could be excluded. Further, these experiments demonstrate that the nature of the induced structures is also observed in intact cells. Another recent study that was using protoplast transfection instead of Agrobacterium-infiltration of leaves observed similar structures when transfecting cells with various

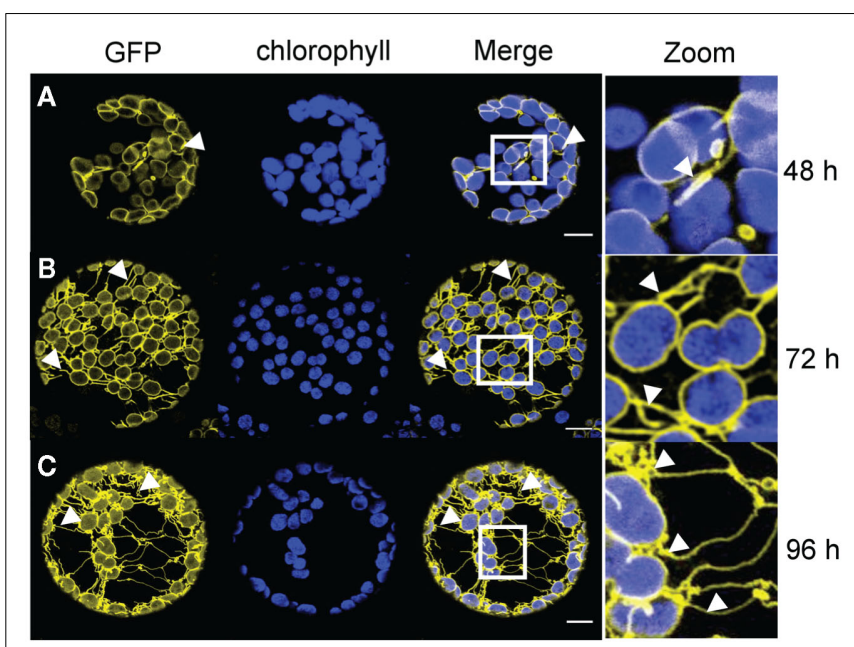

FIGURE 5 | Time course analysis of expression patterns for AtLACS9-GFP in $\boldsymbol{N}$. benthamiana protoplasts. Time based confocal microscopic analysis on isolated protoplasts of $N$. benthamiana leaves after 35 S-promoter driven in planta expression of AtLACS9-GFP. GFP fluorescence (GFP) in yellow, chlorophyll autofluorescence (chlorophyll) in blue, an overlay (merge) and a magnification (zoom) of representative protoplasts are shown. The white box indicates the merged region magnified under zoom. (A) Expression pattern 2 days (48h) after Agrobacterium infiltration shows ring structures surrounding the chloroplasts with slight extensions proliferating from the ring. (B) Expression pattern 3 days ( $72 \mathrm{~h}$ ) after Agrobacterium infiltration displays more pronounced extensions in addition to ring like structures surrounding the chloroplasts. (C) Expression pattern 4 days (96 h) after Agrobacterium infiltration shows a network of GFP labeled structures surrounding the chloroplasts, spanning though the entire protoplasts and connecting chloroplasts. Size bars: $10 \mu \mathrm{m}$. 
tagged inner envelope and outer envelope proteins (Machettira et al., 2012).

The expression of an untagged AtLACS9 led to the formation of tubular structures protruding from the chloroplast (Figure 7). This demonstrated that the membrane alteration is not due to the GFP-fusion. We achieved similar results to those described above with constructs driven by the ubiquitin 10 promoter and $\beta$ estradiol inducible promoter (data not shown). Thus, the observed alterations were not due to an effect of the $35 \mathrm{~S}$ cauliflower mosaic virus promoter.

\section{INVESTIGATING THE IDENTITY OF STRUCTURAL ALTERATIONS}

The tubular structures we observed during expression of the outer envelope proteins AtTOC64 and AtLACS9 were reminiscent of the previously described stromules (e.g., Köhler and Hanson, 2000; Hanson and Köhler, 2001). Stromules are stroma filled extensions
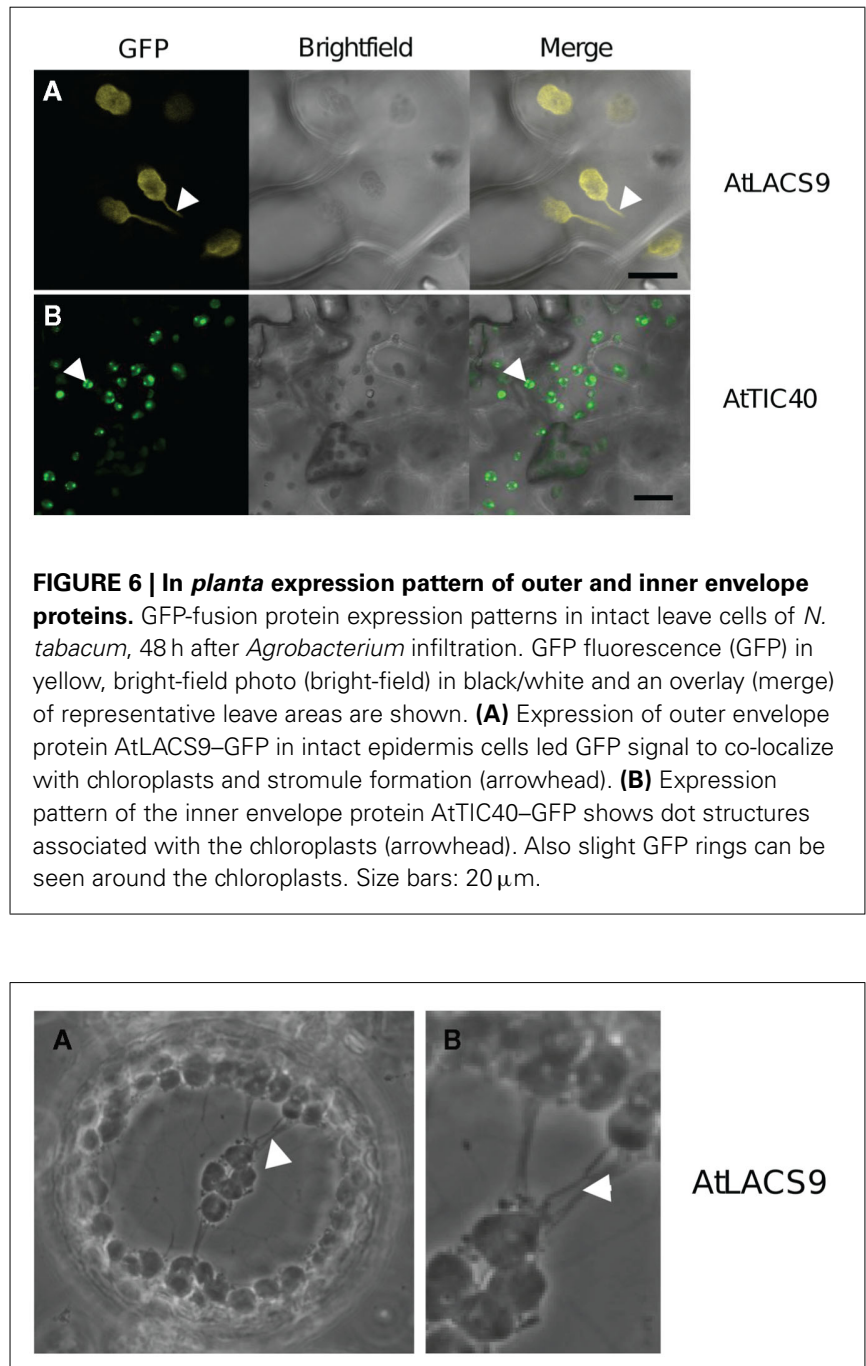

FIGURE 7 | AtLACS9 $\mathbf{w} / 0$ GFP expression in $\boldsymbol{N}$. benthamiana leaf protoplast. Protoplast of a transfected $N$. benthamiana leaf cell expressing AtLACS9 driven by a $35 \mathrm{~S}$-promoter. (A) Chloroplasts are connected by a system of strings (arrowhead). (B) Close-up of the connections between the chloroplasts. from plastids that are surrounded by the inner and outer envelope membranes. Previous work on the inner envelope protein AtTIC40 reported similar structures compared to those observed in our study and it was concluded that the inner envelope proliferated in several layers when expressing this protein from the chloroplast genome. It had also been shown that the outer envelope was not affected by these proliferations (Singh et al., 2008).

To further investigate the induced structures, we performed transmission electron microscopy on transiently transformed $N$. benthamiana leaves, expressing AtLACS9-GFP or AtTIC40-GFP. The analysis showed that chloroplast of transfected cells expressing AtLACS9 had extensions filled with stroma that were surrounded by two membranes (Figures $\mathbf{8 A - C}$ ), the inner and the outer envelope. We further observed direct connections between distinct chloroplasts via such stroma filled extensions (Figure $\mathbf{8 A}$ and insert). In addition, organelles like mitochondria (Figure 8B) and

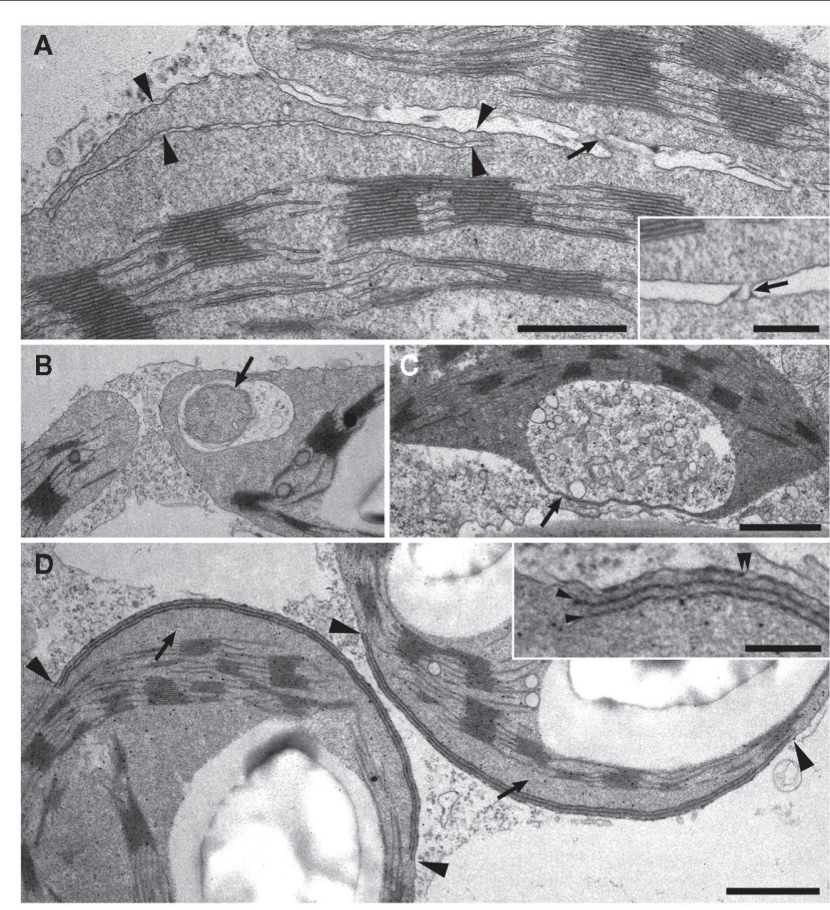

FIGURE 8 | Analysis of transient transfected $\boldsymbol{N}$. benthamiana leave cells by transmission electron microscopy. Ultrastructural analysis of leave material of $N$. benthamiana plants transiently transfected with the constructs 35S:AtLACS9-GFP (A-C) or 35S:AtTIC40-GFP (D). [(A); and inset] Stroma filled connection sites (arrows) between chloroplasts in AtLACS9 transfected tobacco cells. The lower chloroplast displays a thin, stroma filled extension (arrowheads). Scale bar in (A) $0.5 \mu \mathrm{m}$, scale bar in the inset: $0.2 \mu \mathrm{m}$. (B) An AtLACS9-GFP containing chloroplast enclosing a mitochondrion (arrow) by a stroma filled extension. (C) A chloroplast of an AtLACS9-GFP expressing tobacco cell displaying a very thin, stroma filled tube. Scale bar in (C) [applies also to (B)]: $1 \mu \mathrm{m}$. [(D) and inset] Chloroplasts of AtTIC40-GFP transfected tobacco cells displaying areas with layers of inner envelope membrane (between arrowheads). The inset shows a higher magnification of additional layers of inner envelope membrane (arrowheads). A double arrowhead labels the outer and inner envelope membranes in close proximity. The proliferated membrane withdraws from the thylakoid membrane and generates a thylakoid free area (arrows). Scale bar in (D) $1 \mu \mathrm{m}$, scale bar in the inset: $0.25 \mu \mathrm{m}$. 
peroxisomes appeared to be surrounded by stroma filled loops of the chloroplast (Figure 8C). These latter observations were comparable with ultrastructural analyses of rice leaves (Sage and Sage, 2009). We concluded that the structures observed by confocal microscopy were stromules, because they contain stroma and are surrounded by the inner and outer envelope membranes (Köhler and Hanson, 2000).

The structures observed in AtTIC40-transfected cells were remarkably different from those of the outer envelope proteintransfected cells. Additional membrane layers were observed in distinct areas (Figure 8D and insert). These proliferations affected only a part of the inner envelope, while the majority of the inner envelope surface appeared unaffected and did not proliferate. These results are consistent with a previous study in which AtTIC40 was expressed from the chloroplast genome and lead to proliferation of inner envelope membrane (Singh et al., 2008). In addition to the proliferations in distinct areas, the folded membrane with drew from the chloroplast center creating a thylakoid free area (Figure 8D). These effects could also be seen in confocal microscopy pictures, where the GFP signal the chlorophyll autofluorescence were separated by a gap (Figure 2A zoom).

The results of the ultrastructural analysis by transmission electron microscopy indicated that the inner and outer envelope membranes reacted differently to over-expression of some of their protein constituents. In the case of the inner envelope membrane, membrane proliferations were formed that extended in several layers into the stroma without affecting the outer envelope membrane. In the case of the outer envelope membrane, membrane proliferation led to the formation of stromule-like structures that contained both the inner and the outer envelope membrane. Thus, proliferation of the outer envelope membrane was accompanied by a simultaneous proliferation of the inner envelope membrane.

\section{DISCUSSION}

In this study we showed that envelope membranes reproducibly proliferate in distinct patterns as increasing amounts of proteins are inserted into the membranes due to over-expression of envelope membrane proteins. We suggest that the structures observed after over-expression of envelope membrane proteins were indicative of the localization of the protein to either the inner or the outer envelope membrane, thereby providing a tool for assessing the localization of unknown proteins. Using a set of different inner envelope proteins we demonstrated that the proliferation of the inner envelope previously described by Singh et al. (2008), who expressed AtTic40 from the chloroplast genome, could also be achieved by nuclear expression and was likely due to the protein amount in the membrane and not to the protein function or structure.

Our work localized the protein AtLACS9 to the chloroplast outer envelope. We were able to show that AtLACS9 indeed labeled stromules in support of previous observations (Schnurr et al., 2002). Several plant species, such as rice (Sage and Sage, 2009) and the arctic-alpine plant Oxyria digyna (Gunning, 2005; Holzinger et al., 2007a,b) contain stromule-like structures in their mesophyll cells. However, the formation of stromules was shown to be much more frequent in non-green tissues (Köhler and Hanson, 2000; Pyke and Howells, 2002; Waters et al., 2004; Natesan et al.,
2005; Hanson and Sattarzadeh, 2008). Here, we show that stromule formation can also be induced by over-expression of outer envelope proteins. Using the outer envelope proteins AtLACS9 and AtTOC64-III, we were able to show that the formation is independent of the function or structure of the protein. Hence we posit that the proliferation of the membrane was a direct effect of the protein content in the membrane, as outlined above for inner envelope proteins. Furthermore, we report that inner and outer envelope membrane was differentially affected by overexpression of their protein constituents. This may also indicate that the membrane structures were controlled by different regulatory mechanisms. Similar results have been recently obtained using transiently transfected protoplasts (Machettira et al., 2012).

\section{STROMULES - CONTROLLED PLASTID SHAPE LOSS}

It is debated whether filament-forming proteins, such as the stroma-localized plastid division protein FtsZ and the cytosolic actin filaments are involved in plastid shape maintenance and the formation of stromules (Kwok and Hanson, 2003; Hanson and Sattarzadeh, 2008, 2011; Martin et al., 2009; Natesan et al., 2009; Reski, 2009; Sattarzadeh et al., 2009). It can be assumed that the inner envelope membrane is the surface for these plastid internal structural elements, while the outer envelope is the surface for interaction with the cytoskeleton.

The withdrawal of the folded inner envelope from the chloroplast center (Figure 8D) represented a shape loss of the organelle, a departure from the usually stable lens shape. Withdrawal of the folded up inner envelope from the thylakoid was observed in both electron micrographs (Figure 8D) and live cell imaging (Figure 2A zoom), which indicated that it was not a preparation artifact. If there is indeed an inner "plastoskeleton" as suggested (compare Reski, 2009; Hanson and Sattarzadeh, 2011), the loss of the normal shape of the chloroplast might be related to a loss of connection sides between the plastoskeleton and the membrane. The membrane populations of the thylakoids and the inner envelope not only remained separate; they were spaced even further apart upon proliferation. Excess inner envelope membrane did not traffic toward the thylakoids (Figure 8D). The outer envelope did not proliferate in concert with the inner envelope (Figure 8D). Two alternative hypotheses explaining these observations could be envisaged: (i) limiting membrane amount of the outer envelope limited membrane expansion and thus shape loss. (ii) Alternatively, either an internal or external skeleton held plastid shape constant, thus forcing the inner envelope to proliferate in layers.

Observing the outer envelope allows differentiation between these hypotheses. The outer envelope membrane proliferated in the form of stromule-like extensions, a different kind of shape loss of the chloroplast. Since both over-expression of inner and of outer envelope proteins promoted plastid shape loss, albeit different types, it was not the limited membrane amount which maintained plastid shape. We were able to show that in the plastid extensions the inner envelope and stroma were present. It was previously proposed that the formation of stromules is due to an interaction of internal pressure of the chloroplast and/or an external pulling by the cytoskeleton (compare Hanson and Sattarzadeh, 2011). Starting from this premise, the proliferation of the outer membrane induces or even forces pulling from an extra-plastidial 
component. Presumably, the proliferation of outer envelope causes the loss of a stabilizing factor residing on the cytosolic side. Following this model, inner envelopes proliferated to accommodate stromule formation induced by the outer envelope. This hypothesis leads to the question of whether the external factors involved in maintaining plastid shape provide the majority of chloroplast stability. Such cytosolic factors might provide the driving force of stromule formation under natural conditions.

\section{IMPLICATION FOR FUTURE RESEARCH}

The results presented here allow localization of envelope proteins and provide new observations on stromule formation. The differential response of the inner and the outer envelope may also provide a tool to investigate the regulation of envelope formation. Singh et al. (2008) observed the same structures as reported here in stably transformed N. tabacum plants over-expressing the Tic40 protein from the chloroplast genome. The insertion of the protein expressed from the chloroplast genome is independent of the TIC/TOC translocon complexes and possibly represents the reason why inner envelope proliferation was never reported for stable expression from the nuclear genome. This would indicate that the TIC/TOC complex is actively involved in the regulation of membrane protein concentration and indirectly prevents the membrane from accumulating excess protein amounts. The protein load appears to be the signal for the plant to commit additional membrane lipids to the inner envelope. Singh et al. (2008) observed increased amounts of other inner envelope proteins when over-expressing the Tic40 protein from the chloroplast, but no increase in outer envelope proteins. Since we found that outer envelope proliferation is accompanied by a proliferation of the inner envelope (Figures $\mathbf{8 A}-\mathbf{C}$ ), we speculate that the proliferation of the outer membrane is associated with increased inner envelope protein levels. Altered protein levels of nuclear

\section{REFERENCES}

Arimura, S., Hirai, A., and Tsutsumi, N. (2001). Numerous and highly developed tubular projections from plastids observed in tobacco epidermal cells. Plant Sci. 160, 449-454.

Bae, W., Lee, Y. J., Kim, D. H., Lee, J., Kim, S., Sohn, E. J., and Hwang, I. (2008). AKr2A-mediated import of chloroplast outer membrane proteins is essential for chloroplast biogenesis. Nat. Cell Biol. 10, 220-227.

Batoko, H., Zheng, H. Q., Hawes, C., and Moore, I. (2000). A Rab1 GTPase is required for transport between the endoplasmic reticulum and Golgi apparatus and for normal Golgi movement in plants. Plant Cell 12, 2201-2217.

Benning, C. (2009). Mechanisms of lipid transport involved in organelle biogenesis in plant cells. Annu. Rev. Cell Dev. Biol. 25, 71-91.

Bionda, T., Tillmann, B., Simm, S., Beilstein, K., Ruprecht, M., and Schleiff, E. (2010). Chloroplast import signals: the length requirement for

encoded proteins indicate that the protein load in the plastid envelope is communicated to the nucleus (Singh et al., 2008). Recently, a chloroplast envelope protein was found that is proposed to be involved in the signaling between chloroplast and nucleus (Sun et al., 2011). Investigating the expression levels of this protein or similar proteins while over-expressing outer or inner envelope proteins might pinpoint possible regulators involved in the regulation of protein abundance in envelope membranes.

Our observations on the proliferation of the inner envelope membrane and the outer envelope membrane (Figure 8) raises the question of whether the proliferation is only due to increased protein amount or also due to an increase in lipid content of the membrane. Unlike the plastid envelopes, the mitochondrial inner membrane (Guidotti, 1972) and the thylakoid membranes (Block et al., 1983) are described as naturally protein dense membrane structures of organelles. Both membranes are either folded (thylakoids; Hodge et al., 1955) or display out-looping formations (mitochondrial inner membrane; Palade, 1953). These structures are similar to the observations for the inner and outer envelope of plastids when over-expressing their respective proteins. Therefore, an increase of membrane lipid production would not necessarily be a prerequisite for proliferating the membrane systems. Analyzing the protein to (plastid-) lipid ratio in inducible stable lines before and after induction of membrane proliferation could answer that question.

\section{ACKNOWLEDGMENTS}

This work was funded by a grant of the Deutsche Forschungsgemeinschaft (WE2231/4-1 to APMW) in the framework of the Arabidopsis Functional Genomics Network (AFGN).We thank the German-American Fulbright Commission, Alisandra Denton, and Sebastian Breuers for supporting this work in diverse ways.

M. (2008b). Comparison of the use of a species-specific database generated by pyrosequencing with databases from related species for proteome analysis of pea chloroplast envelopes. J. Biotechnol. 136, 44-53.

Bräutigam, A., and Weber, A. P. M. (2009). Proteomic analysis of the proplastid envelope membrane provides novel insights into small molecule and protein transport across proplastid membranes. Mol. Plant 2, 1247-1261.

Breuers, F. K. H., Bräutigam, A., and Weber, A. P. M. (2011). The plastid outer envelope - a highly dynamic interface between plastid and cytoplasm. Front. Plant Sci. 2:97. doi:10.3389/fpls.2011.00097

Chou, M.-L. (2003). Tic40, a membrane-anchored co-chaperone homolog in the chloroplast protein translocon. EMBO J. 22, 10.

Curtis, M. D., and Grossniklaus, U. (2003). A gateway cloning vector set for high-throughput functional analysis of genes in planta. Plant Physiol. 133, 462-469.
Dreses-Werringloer, U., Fischer, K., Wachter, E., Link, T. A., and Flugge, U. I. (1991). cDNA sequence and deduced amino-acid-sequence of the precursor of the $37-\mathrm{Kda}$ inner envelope membrane polypeptide from spinach-chloroplasts - its transit peptide contains an amphiphilic alpha-helix as the only detectable structural element. Eur. J. Biochem. 195, 361-368.

Facchinelli, F., and Weber, A. P. M. (2011). The metabolite transporters of the plastid envelope: an update. Front. Plant Sci. 2:50. doi:10.3389/fpls.2011.00050

Ferro, M., Brugiere, S., Salvi, D., Seigneurin-Berny, D., Court, M., Moyet, L., Ramus, C., Miras, S., Mellal, M., Le Gall, S., Kieffer-Jaquinod, S., Bruley, C., Garin, J., Joyard, J., Masselon, C., and Rolland, N. (2010). AT_CHLORO, a comprehensive chloroplast proteome database with subplastidial localization and curated information on envelope proteins. Mol. Cell. Proteomics 9, 1063-1084. 
Ferro, M., Salvi, D., Brugiere, S., Miras, S., Kowalski, S., Louwagie, M., Garin, J., Joyard, J., and Rolland, N. (2003). Proteomics of the chloroplast envelope membranes from Arabidopsis thaliana. Mol. Cell. Proteomics 2, 325-345

Fischer, K., Arbinger, B., Kammerer, B., Busch, C., Brink, S., Wallmeier, H., Sauer, N., Eckerskorn, C., and Flugge, U. I. (1994). Cloning and in-vivo expression of functional triose phosphate/phosphate translocators from C-3-plants and C-4plants - evidence for the putative participation of specific aminoacid-residues in the recognition of phosphoenolpyruvate. Plant J. 5, 215-226.

Gehl, C., Waadt, R., Kudla, J., Mendel, R. R., and Hansch, R. (2009). New GATEWAY vectors for high throughput analyses of proteinprotein interactions by bimolecular fluorescence complementation. Mol. Plant 2, 1051-1058.

Grefen, C., Donald, N., Hashimoto, K., Kudla, J., Schumacher, K., and Blatt, M. R. (2010). A ubiquitin-10 promoter-based vector set for fluorescent protein tagging facilitates temporal stability and native protein distribution in transient and stable expression studies. Plant J. 64, 355-365.

Guidotti, G. (1972). Membrane proteins. Annu. Rev. Biochem. 41, 731-752.

Gunning, B. E. S. (2005). Plastid stromu les: video microscopy of their outgrowth, retraction, tensioning, anch oring, branching, bridging, and tipshedding. Protoplasma 225, 33-42.

Hanson, M. R., and Köhler, R. H. (2001). GFP imaging: methodology and application to investigate cellular compartmentation in plants. $J$. Exp. Bot. 52, 529-539.

Hanson, M. R., and Sattarzadeh, A. (2008). Dynamic morphology of plastids and stromules in angiosperm plants. Plant Cell Environ. 31, 646-657.

Hanson, M. R., and Sattarzadeh, A. (2011). Stromules: recent insights into a long neglected feature of plastid morphology and function. Plant Physiol. 155, 1486-1492.

Hodge, A. J., Mclean, J. D., and Mercer, F. V. (1955). Ultrastructure of the lamellae and grana in the chloroplast of Zea Mays L. J. Biophys. Biochem. Cytol. 1, 605

Holzinger, A., Buchner, O., Lutz, C., and Hanson, M. R. (2007a). Temperature-sensitive formation of chloroplast protrusions and stromules in mesophyll cells of
Arabidopsis thaliana. Protoplasma 230, 23-30.

Holzinger, A., Wasteneys, G. O., and Lutz, C. (2007b). Investigating cytoskeletal function in chloroplast protrusion formation in the arcticalpine plant Oxyria digyna. Plant Biol. 9, 400-410.

Joyard, J., Ferro, M., Masselon, C., Seigneurin-Berny, D., Salvi, D., Garin, J., and Rolland, N. (2010). Chloroplast proteomics highlights the subcellular compartmentation of lipid metabolism. Prog. Lipid Res. $49,128-158$.

Köhler, R., Hanson, M., and Wildman, S. (1997a). Pictures in cell biology - plastid interconnections imaged by fluorescence and phase contrast. Trends Cell Biol. 7, 392-392.

Köhler, R. H., Cao, J., Zipfel, W. R., Webb, W. W., and Hanson, M. R. (1997b). Exchange of protein molecules through connections between higher plant plastids. Science 276, 2039-2042.

Köhler, R. H., and Hanson, M. R. (2000). Plastid tubules of higher plants are tissue-specific and developmentally regulated. J. Cell Sci. 113, 81-89.

Koncz, C., and Schell, J. (1986). The promoter of Tl-DNA gene 5 controls the tissue-specific expression of chimeric genes carried by a novel type of Agrobacterium binary vector. Mol. Gen. Genet. 204, 383-396.

Koo, A. J., Ohlrogge, J. B., and Pollard, M. (2004). On the export of fatty acids from the chloroplast. J. Biol. Chem. 279, 16101-16110.

Kwok, E. Y., and Hanson, M. R. (2003). Microfilaments and microtubules control the morphology and movement of non-green plastids and stromules in Nicotiana tabacum. Plant J. $35,16-26$.

Lee, Y. J., Kim, D. H., Kim, Y. W., and Hwang, I. (2001). Identification of a signal that distinguishes between the chloroplast outer envelope membrane and the endomembrane system in vivo. Plant Cell 13, 2175-2190.

Linka, N., and Weber, A. P. M. (2010). Intracellular metabolite transporters in plants. Mol. Plant 3, 21-53.

Machettira, A. B., Groß, L. E., Tillmann, B., Weis, B. L., Englich, G., Sommer, M. S., Königer, M., and Schleiff, E. (2012). Protein induced modulation of chloroplast membrane morphology. Front. Plant Sci. 2:118. doi: 10.3389/fpls.2011.00118

Martin, A., Lang, D., Hanke, S. T., Mueller, S. J. X., Sarnighausen, E., Vervliet-Scheebaum, M., and Reski, R. (2009). Targeted gene knockouts reveal overlapping functions of the five Physcomitrella patens FtsZ isoforms in chloroplast division, chloroplast shaping, cell patterning, plant development, and gravity sensing. Mol. Plant 2, 1359-1372.

Motohashi, R., Ito, T., Kobayashi, M., Taji, T., Nagata, N., Asami, T., Yoshida, S., Yamaguchi-Shinozaki, K., and Shinozaki, K. (2003). Functional analysis of the $37 \mathrm{kDa}$ inner envelope membrane polypeptide in chloroplast biogenesis using a Ds-tagged Arabidopsis pale-green mutant. Plant J. 34, 719-731.

Natesan, S. K. A., Sullivan, J. A., and Gray, J. C. (2005). Stromules: a characteristic cell-specific feature of plastid morphology. J. Exp. Bot. 56, 787-797.

Natesan, S. K. A., Sullivan, J. A., and Gray, J. C. (2009). Myosin XI is required for actin-associated movement of plastid stromules. Mol. Plant 2, 1262-1272.

Oikawa, K., Kasahara, M., Kiyosue, T., Kagawa, T., Suetsugu, N., Takahashi, F., Kanegae, T., Niwa, Y., Kadota, A., and Wada, M. (2003). Chloroplast unusual positioning1 is essential for proper chloroplast positioning. Plant Cell 15, 2805-2815.

Oikawa, K., Yamasato, A., Kong, S. G., Kasahara, M., Nakai, M., Takahashi, F., Ogura, Y., Kagawa, T., and Wada, M. (2008). Chloroplast outer envelope protein CHUP1 is essential for chloroplast anchorage to the plasma membrane and chloroplast movement. Plant Physiol. 148, 829-842.

Palade, G. E. (1953). An electron microscope study of the mitochondrial structure. J. Histochem. Cytochem. 1 , 188-211.

Pyke, K. A., and Howells, C. A. (2002). Plastid and stromule morphogenesis in tomato. Ann. Bot. 90, 559-566.

Qbadou, S., Becker, T., Bionda, T., Reger, K., Ruprecht, M., Soll, J., and Schleiff, E. (2007). Toc64 - A preproteinreceptor at the outer membrane with bipartide function. J. Mol. Biol. 367, 1330-1346.

Reski, R. (2009). Challenges to our current view on chloroplasts. Biol. Chem. 390, 731-738.

Reynolds, E. S. (1963). Use of lead citrate at high $\mathrm{pH}$ as an electronopaque stain in electron microscopy. J. Cell Biol. 17, 208-212.

Rolland, N., Ferro, M., SeigneurinBerny, D., Garin, J., Douce, R. and Joyard, J. (2003). Proteomics of chloroplast envelope membranes. Photosyn. Res. 78, 205-230.

Roughan, P. G., and Slack, C. R. (1977). Long-chain acyl-coenzyme A synthetase activity of spinach chloroplasts is concentrated in the envelope. Biochem. J. 162, 457-459.

Sage, T. L., and Sage, R. F. (2009). The functional anatomy of rice leaves: implications for refixation of photorespiratory $\mathrm{CO} 2$ and efforts to engineer C-4 photosynthesis into rice. Plant Cell Physiol. 50, 756-772.

Sattarzadeh, A., Krahmer, J., Germain, A. D., and Hanson, M. R. (2009). A Myosin XI tail domain homologous to the yeast myosin vacuolebinding domain interacts with plastids and stromules in Nicotiana benthamiana. Mol. Plant 2, 1351-1358.

Schmidt Von Braun, S., and Schleiff, E. (2008). The chloroplast outer membrane protein CHUP1 interacts with actin and profilin. Planta 227, 1151-1159.

Schnurr, J. A., Shockey, J. M., De Boer, G. J., and Browse, J. A. (2002). Fatty acid export from the chloroplast. Molecular characterization of a major plastidial acyl-coenzyme A synthetase from Arabidopsis. Plant Physiol. 129, 1700-1709.

Shaw, D. J., and Gray, J. C. (2011). Visualisation of stromules in transgenic wheat expressing a plastid-targeted yellow fluorescent protein. Planta 233, 961-970.

Shiina, T., Hayashi, K., Ishii, N., Morikawa, K., and Toyoshima, Y. (2000). Chloroplast tubules visualized in transplastomic plants expressing green fluorescent protein. Plant Cell Physiol. 41, 367-371.

Shockey, J. M., Fulda, M. S., and Browse, J. A. (2002). Arabidopsis contains nine long-chain acyl-coenzyme A synthetase genes that participate in fatty acid and glycerolipid metabolism. Plant Physiol. 129, 1710-1722.

Singh, N. D., Li, M., Lee, S. B., Schnell, D., and Daniell, H. (2008). Arabidopsis Tic40 expression in tobacco chloroplasts results in massive proliferation of the Inner envelope membrane and upregulation of associated proteins. Plant Cell 20, 3405-3417.

Sohrt, K., and Soll, J. (2000). Toc64, a new component of the protein translocon of chloroplasts. J. Cell Biol. 148, 1213-1221.

Strittmatter, P., Soll, J., and Bolter, B. (2010). The chloroplast protein import machinery: a review. Methods Mol. Biol. 619, 307-321.

Sun, Q., Zybailov, B., Majeran, W., Friso, G., Olinares, P. D., and Van Wijk, K. J. (2009). PPDB, the plant proteomics database at Cornell. Nucleic Acids Res. 37, D969-D974.

Sun, X., Feng, P., Xu, X., Guo, H., Ma, J., Chi, W., Lin, R., Lu, C., 
and Zhang, L. (2011). A chloroplast envelope-bound PHD transcription factor mediates chloroplast signals to the nucleus. Nat. Commun. 2, 477.

Tirlapur, U. K., Dahse, I., Reiss, B., Meurer, J., and Oelmuller, R. (1999). Characterization of the activity of a plastid-targeted green fluorescent protein in Arabidopsis. Eur. J. Cell Biol. 78, 233-240.

Voinnet, O., Rivas, S., Mestre, P., and Baulcombe, D. (2003). An enhanced transient expression system in plants based on suppression of gene silencing by the p19 protein of tomato bushy stunt virus. Plant J. 33, 949-956.

Waters, M. T., Fray, R. G., and Pyke, K. A. (2004). Stromule formation is dependent upon plastid size, plastid differentiation status and the density of plastids within the cell. Plant J.39, 655-667.
Weber, A. P. M., and Linka, N. (2011). Connecting the plastid: transporters of the plastid envelope and their role in linking plastidial with cytosolic metabolism. Annu. Rev. Plant Biol. 62, 53-77.

Wydro, M., Kozubek, E., and Lehmann, P. (2006). Optimization of transient Agrobacterium-mediated gene expression system in leaves of Nicotiana benthamiana. Acta Biochim. Pol. 53, 289-298.

Yang, Y., Jin, H., Chen, Y., Lin, W., Wang, C., Chen, Z., Han, N., Bian, H., Zhu, M., and Wang, J. (2012). A chloroplast envelope membrane protein containing a putative $\operatorname{LrgB}$ domain related to the control of bacterial death and lysis is required for chloroplast development in Arabidopsis thaliana. New Phytol. 193, 81-95.
Yoo, S. D., Cho, Y. H., and Sheen, J. (2007). Arabidopsis mesophyll protoplasts: a versatile cell system for transient gene expression analysis. Nat. Protoc. 2, 1565-1572.

Zhao, L., Katavic, V., Li, F., Haughn, G. W., and Kunst, L. (2010). Insertional mutant analysis reveals that long-chain acyl-CoA synthetase 1 (LACS1), but not LACS8, functionally overlaps with LACS9 in Arabidopsis seed oil biosynthesis. Plant J. 64 1048-1058.

Conflict of Interest Statement: The authors declare that the research was conducted in the absence of any commercial or financial relationships that could be construed as a potential conflict of interest.
Received: 26 October 2011; accepted: 05 January 2012; published online: 23 January 2012.

Citation: Breuers FKH, Bräutigam A, Geimer S, Welzel UY, Stefano G, Renna L, Brandizzi $F$ and Weber APM (2012) Dynamic remodeling of the plastid envelope membranes - a tool for chloroplast envelope in vivo localizations. Front. Plant Sci. 3:7. doi: 10.3389/fpls.2012.00007

This article was submitted to Frontiers in Technical Advances in Plant Science, a specialty of Frontiers in Plant Science. Copyright (c) 2012 Breuers, Bräutigam, Geimer, Welzel, Stefano, Renna, Brandizzi and Weber. This is an open-acces article distributed under the terms of the Creative Commons Attribution Non Commercial License, which permits noncommercial use, distribution, and reproduction in other forums, provided the original authors and source are credited. 